\title{
The Auger Raman Lidar: several years of continuous observations
}

\author{
Vincenzo Rizi $^{1 *}$ for The Pierre Auger Collaboration ${ }^{2, * *, * * *}$, Carlos Medina Hernandez ${ }^{3}$, Marco Iarlori ${ }^{1}$ and Ermanno \\ Pietropaolo ${ }^{1}$.
}

${ }^{1}$ INFN-GSGC - L'Aquila and CETEMPS/DSFC, Università Degli Studi dell'Aquila, L'Aquila, Italy.

${ }^{2}$ Observatorio Pierre Auger, Av. San Martín Norte 304, 5613 Malargüe, Argentina.

${ }^{3}$ Department of Physics, Colorado, School of Mines, Golden, Colorado, USA.

\begin{abstract}
The Raman lidar at the Central (Raman) Laser Facility of the Pierre Auger Observatory in Argentina, has been operational since September 2013. In this paper, the Auger Raman Lidar performance is discussed in terms of the data quality for the assessment of the aerosol contribution to the atmospheric UV optical transparency, and how much this is important for the reconstruction of the UHECR properties, based on the Auger Fluorescence Detector observations.
\end{abstract}

\section{INTRODUCTION}

The Pierre Auger Observatory (Auger) [1] in Malargüe, Argentina, is designed to study the properties of ultrahigh energy cosmic rays (UHECR) with energies above $10^{18} \mathrm{eV}$, using the terrestrial atmosphere as an enormous calorimeter. Auger uses Fluorescence Detectors (FD) to perform nearly calorimetric measurements of Extensive Air Shower (EAS) energies. To obtain reliable calorimetric information from the FD, the atmospheric conditions at the observatory need to be continuously monitored during data acquisition: a cosmic ray EAS generates locally UV fluorescent light in proportion to the energy deposited; the amount of UV light reaching the FD at ground depends on the atmospheric optical transparency. In particular, light attenuation due to aerosols is an important atmospheric correction. The atmospheric Vertical Aerosol Optical Depth (VAOD), although smaller than the corresponding optical depth profile of the molecular component, can vary significantly on short time scales. The aerosol concentration is highly variable, so that the aerosol attenuation needs to be evaluated hourly.

Auger uses a laser beam emitted from the Central Laser Facility (CLF), located near the center of the Observatory site (see Fig. 1). CLF has an optical signature comparable to that of the highest energy showers detected by the FD, and its laser shots allow measuring the aerosol optical transparency in a portion of the atmosphere between the CLF and the FD sites [2]. A Raman lidar (RL) is also present at CLF, and it measures the VAOD, the aerosol backscatter coefficient, and water vapour mixing ratio profiles on routine basis. In this paper we briefly describe the RL and its performance in measuring the vertical profiles of the aerosol optical properties (section 2).

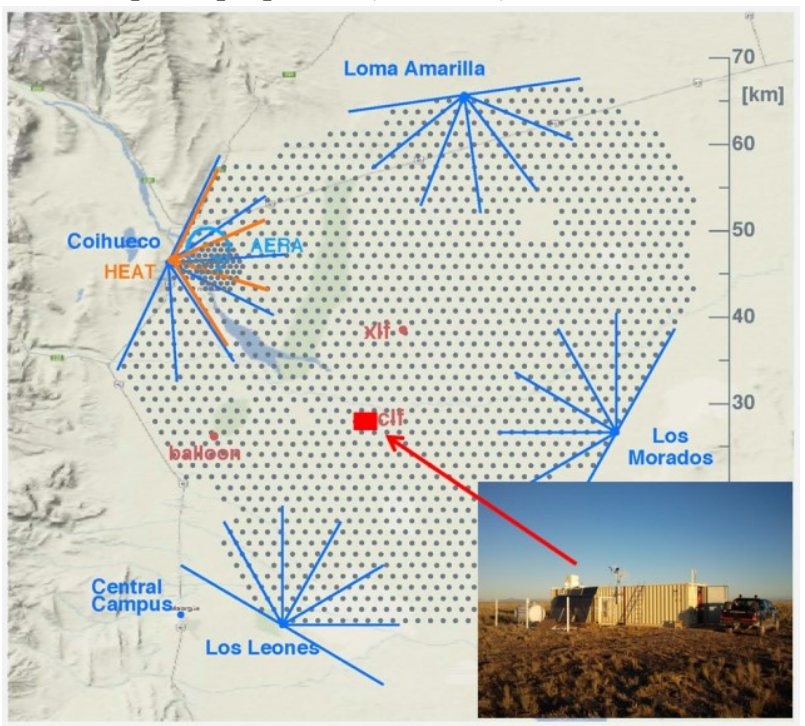

Fig. 1. The Raman Lidar system samples vertically the part of the atmosphere above the CLF site, and the retrieved VAOD profiles allow measuring the aerosol optical transmission in the atmosphere over the Observatory. The red square indicates the position of CLF container (in the picture). The FD telescopes (six for each site) are at Los Leones, Los Morados, Loma Amarilla and Coihueco; the small dots represent the positions of the stations of Surface Detectors (SD) of the Pierre Auger Observatory.

The time-series (data period: 2013-2017) of the relevant parameters are shown in section 3; finally, there is a short discussion of the impact of the aerosol optical properties on the UHECR energy reconstruction.

\footnotetext{
*email: vincenzo.rizi@aquila.infn.it

**email: auger_spokespersons@fnal.gov

***author list: http://www.auger.org/archive/authors_2018_09.html
} 


\section{TECHNICAL DESCRIPTION OF RAMAN LIDAR AND ITS OPERATION AT AUGER}

The RL laser source is a Nd:YAG laser emitting at 354.7 $\mathrm{nm}$, line width about $1 \mathrm{~cm}^{-1}$, pulse duration $7 \mathrm{~ns}$, output energy $6 \mathrm{~mJ}$, divergence $0.3 \mathrm{mrad}$, linearly polarized, repetition rate $100 \mathrm{~Hz}$. The receiving telescope of the Raman lidar consists of a $50 \mathrm{~cm}$ diameter $\mathrm{f} / 3$ parabolic mirror pointing vertically beneath a UV transmitting silica window and a motorized roof hatch. An optical fiber couples the light reflected from the mirror to a three-channel receiver. A combination of dichroic beam splitters and notch filters directs this light onto three photomultiplier tubes (PMTs) that are located behind narrow-band optical filters. They isolate the three scattered wavelengths of interest, $354.7 \mathrm{~nm}$ (Rayleigh/Mie or elastic scattering), $386.7 \mathrm{~nm}$ (Raman $\mathrm{N}_{2}$ backscattering), and $407.5 \mathrm{~nm}$ (Raman $\mathrm{H}_{2} \mathrm{O}$ backscattering). The data acquisition system uses analog (up to $80 \mathrm{MHz} \mathrm{A} / \mathrm{D}$ ) and Photon Counting (PhC, maximum count rate $250 \mathrm{MHz}$ ) acquisition modules. More technical details can be found in [3]. The RL is located in a temperature conditioned container (CLF). Rain and wind sensors conditionally control the opening and closing of the roof hatch.

The RL is automatic and it is run during the FD shift periods in 3 time windows of about 15 minutes Before, During and After (BDA) the daily FD shift: this has a negligible impact on the duty cycles of the FD.

Once a month, the Auger local technical staff is taking care of the regular maintenance of RL at CLF (i.e., cleaning of lenses and mirrors). Yearly, special activities on RL system and sub-systems (i.e., the optical alignments of the laser transmitter and of the receiver bench, and the status of the detectors) are conducted by lidar experts.

In a typical RL run, the elastic lidar signal; the $\mathrm{N}_{2}$ Raman backscatter signal; and the $\mathrm{H}_{2} \mathrm{O}$ Raman lidar return are accumulated and collected for 12 minutes in $\mathrm{A} / \mathrm{D}$ and $\mathrm{PhC}$ mode.

Before starting the signal analysis raw data have to be processed and several operations have to be done:

- to correct the PhC detection for the dead-time effects;

- to subtract the signal due to sky background and the, eventually present, electric noise;

- to set the correct time scale, i.e., the time bin corresponding to the laser pulse emission ( $\left.0^{\text {th }} \mathrm{bin}\right)$;

- to combine the $\mathrm{A} / \mathrm{D}$ and $\mathrm{PhC}$ signals.

The (light) background is evaluated in a region where the atmospheric backscattering is negligible, usually along the first 10 samples or the last 100 samples of the total 1024 of the digitized signals.

The $0^{\text {th }}$ bin position is periodically measured sending directly (with an optical fiber) a very small fraction of the laser pulse in the RL receiver. The signal registered in each RL channel shows a peak in correspondence of the laser emission, and the peak's position indicate the $0^{\text {th }}$ bin time (slightly different from one channel to the other) with a precision of $\pm 25 \mathrm{~ns}$ (using a bin width of $50 \mathrm{~ns}$ or less for the time scale). The correct time scale is obtained subtracting the $0^{\text {th }}$ bin time.
The $\mathrm{A} / \mathrm{D}$ and $\mathrm{PhC}$ signals are binned on the same time (range) scale, and "glued". The "gluing" procedure is the following: the $\mathrm{A} / \mathrm{D}$ and $\mathrm{PhC}$ signals are normalized in a range of altitudes where the $\mathrm{PhC}$ rate is below $10 \mathrm{MHz}$ (lower level) and the A/D signal to noise ratio is higher than 10 (higher level); the final signal is the combination of the $\mathrm{A} / \mathrm{D}$ in the range interval where $\mathrm{PhC}$ is above 10 $\mathrm{Mhz}$, and $\mathrm{PhC}$ signal elsewhere. After applying all these corrections, errors are associated to the signals

\section{MEASUREMENTS OF THE AEROSOL OPTICAL PROPERTIES}

Rayleigh/Mie and Raman lidar inversion methods for the estimation of the aerosol optical properties are well known [4]. The evaluation of the VAOD can be done directly from the $\mathrm{N}_{2}$ Raman Lidar return. The uncertainties affecting the VAOD are the statistical uncertainty due to signal detection, the systematic uncertainty associated with the estimation of the molecular number density and the Rayleigh scattering cross section, the systematic uncertainty associated with the evaluation of the aerosol scattering wavelength dependence (Angstrom coefficient), and the uncertainties introduced by operational procedures such as signal averaging (accumulating lidar returns), and by applying, for example, derivative digital filters. An additional systematic uncertainty that should be accounted for is due to the geometrical overlap function of the lidar. In a range of heights where the optical overlap between the laser and the field of view of the receiving mirror is not complete, this uncertainty can be quite important. The atmospheric temperature and pressure profiles from balloon soundings or global meteorological models (i. e., GDAS) [5] are used to estimate the vertical profile of the air molecular number density, and the Rayleigh scattering components into the Raman lidar returns.

The aerosol volume backscattering coefficient is evaluated starting from the ratio between the elastic and $\mathrm{N}_{2}$ Raman lidar returns. The design of our Raman lidar receiver (the light returns collected by the telescope are transported to the detector box through an optical fiber) assigns the same optical overlap modulation to the Rayleigh/Mie elastic and inelastic $\mathrm{N}_{2}$ Raman lidar channel, a calibration is needed, and usually this is done assuming that there is a range of altitudes free of aerosols, for example in the upper part of the troposphere. The uncertainties affecting the Aerosol Backscatter ( $\mathrm{AB}$ ) profile are mainly due to the statistical uncertainty in the signal detection, the systematic uncertainty associated with the estimation of the molecular number density (i. e., by the vertical profile of the atmospheric molecular number density), the Rayleigh scattering cross section and the uncertainties introduced by operational (retrieval) procedures, such as the calibration.

The water vapour mixing ratio profile is obtained from the ratio between the $\mathrm{H}_{2} \mathrm{O}$ Raman and $\mathrm{N}_{2}$ Raman lidar backscatter signals. A calibration is also needed to include all the system parameters corresponding to the Raman channels. The calibration constant is determined 
from the direct comparisons of the Raman lidar measurements with simultaneous and co-located balloon-borne sensors able to measure the atmospheric water vapour.

In Figure 2, it is reported an example of the results of a single session of measurement, the quantities of interest are the VAOD and $\mathrm{AB}$ vertical profiles.

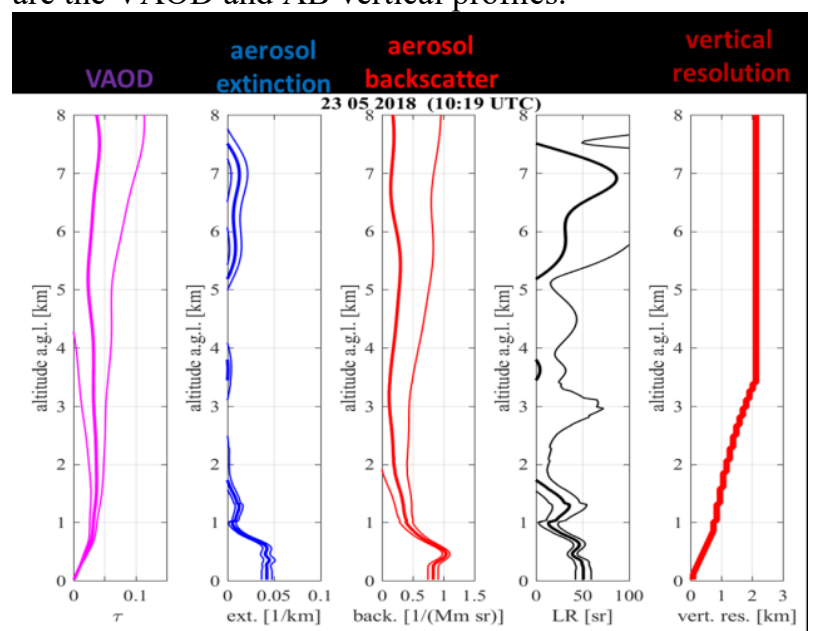

Fig.2. The measured aerosol optical properties in a 12 minutes RL run. From the left to the right panel: the vertical profiles of the aerosol optical depth, its derivative (i.e., Aerosol Extinction, AE), the Aerosol Backscatter coefficient (AB), the Lidar Ratio (LR), that is the ratio $\mathrm{AE} / \mathrm{AB}$, and the vertical resolution of the profiles. The thin lines associated to the profiles (thick lines) give indications of the uncertainty (systematic and uncorrelated errors).

In the range between ground and $3 \mathrm{~km}$ above ground level (agl), the VAOD has a typical uncertainty (uncorrelated + systematics) $\leq 0.005$; the $\mathrm{AB}$ has errors $\leq 0.3 \times 10^{-6} \mathrm{~m}^{-1} \mathrm{sr}^{-1}$, and the AE errors are $\leq 1.0 \times 10^{-5} \mathrm{~m}^{-1}$.
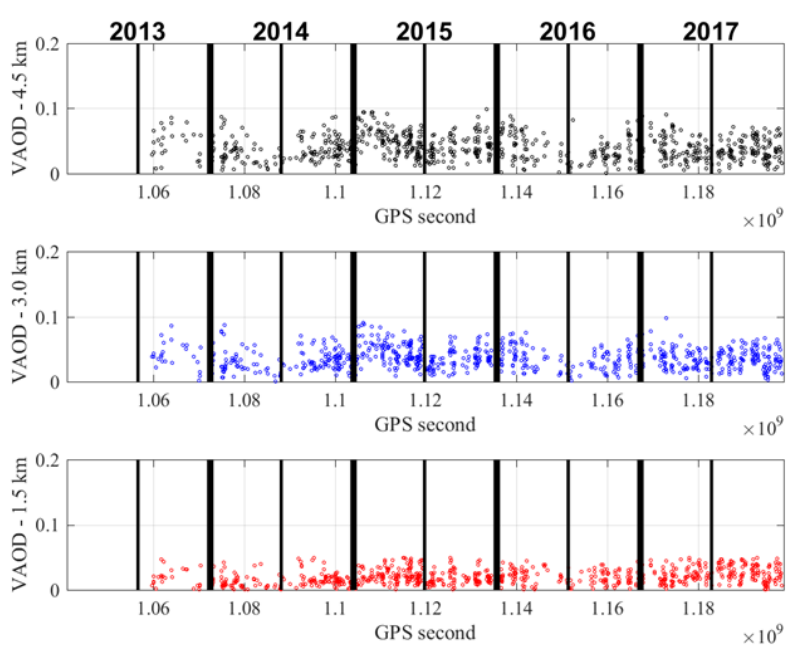

Fig. 3. The VAOD values to $1.5,3.0$ and $4.5 \mathrm{~km}$ agl, in the period September 2013 - December 2017, only the cloud-free cases have been considered.

The Lidar Ratio gives information about the aerosol particle sizes and composition, in the case reported in Figure 2, in the region where most of the aerosols are, i.e., below $1.5 \mathrm{~km}$ agl, the mean LR is about $40 \mathrm{sr}$, a typical value for the aerosol particles in the atmosphere above the Pierre Auger Observatory.
The scheduled measurements have been regularly taken; the RL database cover a period between September 2013 to present. In the period 2013-2017, there are:

○ 2601 measurements of VAOD profile;

- 2488 measurements of the vertical AB profile;

○ 1804 measurements of the vertical $\mathrm{H}_{2} \mathrm{O}$ mixing ratio profile.

The current BDA schedule of the observations has started since October 2014.

A representation of the full set of data is given in Figure 3 , where the VAOD values to $1.5,3.0$ and $4.5 \mathrm{~km}$ agl of the cloud-free profiles ( 807 measurements) are shown as function of the time period: GPS seconds (seconds from 06/01/1980 00:00:00 with leap seconds added). These VAOD values have been calculated averaging the profiles around $( \pm 0.3 \mathrm{~km})$ the corresponding altitude.

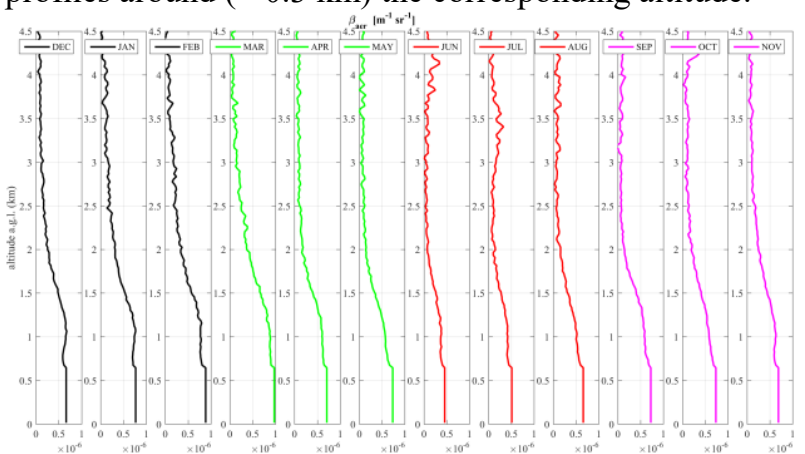

Fig. 4. The monthly mean aerosol backscatter vertical profiles in the period September 2013 - December 2017.

The temporal behaviours of the VAOD values clearly show a seasonal oscillation: higher values in the austral summer and lower ones along the winter.

The mean values and the corresponding standard deviations at $1.5,3.0$ and $4.5 \mathrm{~km}$ agl are $0.020 \pm 0.012$, $0.036 \pm 0.016$ and $0.040 \pm 0.019$, respectively. As expected, the atmosphere over Auger is quite clean.

The monthly mean $\mathrm{AB}$ vertical profiles are shown in Figure 4. The AB profiles show a seasonal dependence of the aerosol load and their vertical distribution. This is clearer if we assume an aerosol model like the one in Figure 5, adopting 3-parameters:

- the PBL height;

- the entrainment zone thickness;

- the aerosol extinction into PBL.

Keeping in mind the equivalence among aerosol content, aerosol backscatter and aerosol extinction coefficient, this kind of model, although quite simplified, accounts for the main features of the aerosol distribution from the PBL to the free troposphere:

- in PBL, because of turbulent dynamics, the aerosol concentration is homogeneously distributed with altitude;

- above there is a transition zone (relatively slow exponential decay of the aerosol extinction coefficient) to the free troposphere where the aerosol content is negligible.

In Figure 4, the monthly mean profiles have a structure quite similar to the 3-parameters model, and following the seasonal cycle: 
- the PBL height is more extended in summer (January, February and March) than in winter (June, July and August);

- the entrainment zone thickness is wider in summer than in winter;

- the load of aerosol into PBL, i.e., high aerosol extinction (or backscatter) coefficient is enhanced in summertime.

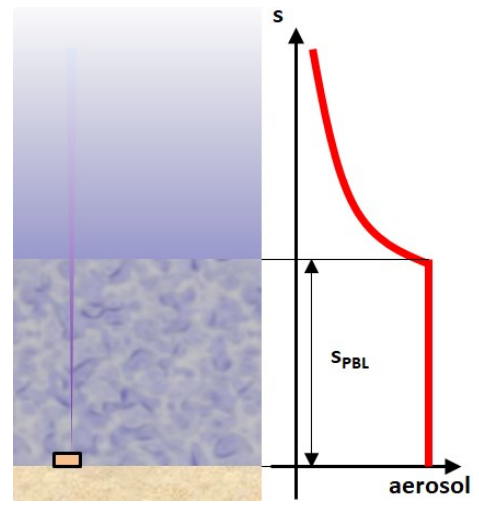

Fig. 5. A 3-parameters aerosol model, $\mathrm{s}$ is the altitude agl for the aerosol extinction:

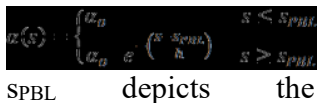

Planetary Boundary Layer (PBL) height and and $h$ the entrainment zone thickness, $\alpha_{o}$ is the aerosol extinction coefficient in the wellmixed PBL.

The performance of this 3-parameters model of the aerosol distribution in the region of the atmosphere above Auger is quite convincing, more details will be discussed elsewhere [8].

\section{DISCUSSION AND CONCLUSIONS}

Can the Raman lidar technique be useful to obtain calorimetric information for the measurements of EAS at Auger? The answer is yes, the Raman lidar has some general advantages with respect to elastic backscatter lidars. A drawback of backscatter lidars is that it is not possible to retrieve the aerosol optical parameters of interest, namely, the aerosol extinction (i.e., attenuation) and the aerosol backscatter (i.e., the mean reflectivity in the observation volume) without introducing correlating hypotheses between these two parameters. This limitation is superseded by the Raman lidar technique, which combine the detection of the elastic backscatter return (Rayleigh scattering of air molecules, and Mie scattering of aerosols) and the inelastic backscatter return (Raman scattering of specific air molecule, i.e., nitrogen or oxygen). The disadvantage of the Raman lidar technique within an observatory like Auger comes from the restriction imposed to not interfere with FD telescope: there is a limited measurement time period, and because of weak Raman scattering cross sections, it needs long data accumulation. Most of the UHECR observatory are situated in sites where the atmosphere is quite clean. The Pierre Auger Observatory is located at about $1400 \mathrm{~m}$ above sea level in the dry pampa region of Argentina. Under these conditions the RL is working at the edge of its sensitivity.

Anyway, the Auger RL is measuring the aerosol optical properties at $355 \mathrm{~nm}$ since November 2013, and:

- the RL data analysis is documented and reproducible;

- the shape of the measured vertical profiles of the aerosol backscatter and extinction suggest a simple model of the aerosol vertical distribution;
- the measured vertical profiles of the aerosol backscatter and extinction show, as expected, a seasonal dependence;

- the database of the raw RL signals, and RL vertical profiles of the aerosol backscatter, VAOD, (and water vapour) will be made available soon with all the documentations and analysis programs.

Finally, the RL at Auger independently measures the vertical profiles of the aerosol optical properties, and it samples a column of the atmosphere located in the central part of Auger; considering this situation, the RL measurements of VAOD can be used as a cross-check for the other technique (namely the CLF method, see [2]) that estimate the aerosol attenuation in different regions of the observatory. A systematic comparison between these two techniques has been conducted in [3], not at Auger, but in a similar setup: there is a systematic small offset, on the other hand the two techniques were well correlated. A deeper analysis within Auger is in progress.

Finally, it is worth recalling the effects that the aerosol optical properties and their vertical distribution have on the EAS energy (E) and $\mathrm{X}_{\max }$ scale reconstructions [6,7]; they are minor contributions; for example, if aerosol attenuation and vertical distribution are completely neglected:

- $X_{\max }$ could show a shift between -1 and $10 \mathrm{~g} / \mathrm{cm}^{2}$, depending on the EAS energy (between $5 \cdot 10^{17} \mathrm{eV}$ and $\left.5 \cdot 10^{19} \mathrm{eV}\right)$;

- the energy scale could be underestimated from $8 \%$, at lower energy, to $25 \%$ at the higher ones.

In the official Auger reconstruction of primary energy and $\mathrm{X}_{\max }$, the VAOD observed with CLF method is used. This contributes to the uncertainties on the energy scale and on $\mathrm{X}_{\max }$ measurements as in the following:

$-\Delta \mathrm{E} / \mathrm{E}<6 \%$;

$-\Delta \mathrm{X}_{\max }<10 \mathrm{~g} / \mathrm{cm}^{2}$.

\section{References}

1. Pierre Auger Collaboration, NIM A, 798, 172-213, (2015)

2. Pierre Auger Collaboration, J. INST., 8, P04009, (2013)

3. L. Wiencke, et al., J. INST., 12, P03008, (2017)

4. I. Mattis, et al., ATMOS. MEAS. TECH., 9, 3009, (2016)

5. Pierre Auger Collaboration, ASTR. PHYS. 35, 591, (2012).

6. Pierre Auger Collaboration, PHYS. REV. D., 90, 122005, (2014).

7. Pierre Auger Collaboration, ASTR. PHYS. 33, 108, (2010).

8. Pierre Auger Collaboration, The Auger Raman lidar, in preparation. 